\title{
Modified Direct and Invers Problem On Triaxial Ellipsoid
}

(Ondokuz Mayis University, Faculty of Engineering, Geomatics Engineering, 55139)

\section{Introduction}

Rotational ellipsoid generally used in geodetic computations. Triaxial ellipsoid surface although a more general so far has not been used in geodetic applications and, the reason for this is not provided as a practical benefit in the calculations. We think this traditional thought ought to be revised again. Today increasing GPS and satellite measurement precision will allow us to determine more realistic earth ellipsoid. Several studies have shown that the Earth, other planets, natural satellites, asteroids and comets can be modeled as triaxial ellipsoids.

It is possible to reduce the most geodesic problems into one of two types. The two geodesic problems usually considered are:

1.1 the direct geodesic problem or first geodesic problem, it is also called coordinate transportation problem. The geographical coordinates of a $\mathrm{P}_{1}$ point $\left(\mathrm{B}_{1}\right.$, $L_{1}$ ), the $S_{12}$ geodesic curve length connecting points $\mathrm{P}_{1}$ and $\mathrm{P}_{2}$ on the triaxial ellipsoid, and $\alpha_{12}$ azimuth on $P_{1}$ at this edge are known. The geographical coordinates of point $\mathrm{P}_{2}\left(\mathrm{~B}_{2}, \mathrm{~L}_{2}\right)$ and the azimuth towards $\alpha_{21}$ are desired, 1.2the inverse geodesic problem or second geodesic problem, It is also called the problem of finding the edge and angle (azimuth). In this basic task, the geographical coordinates of points $\mathrm{P}_{1}$ and $\mathrm{P}_{2}\left(\mathrm{~B}_{1}, \mathrm{~L}_{1}\right)$ and $\left(\mathrm{B}_{2}, \mathrm{~L}_{2}\right)$ are known, the length of the $\mathrm{S}_{12}$ geodesic curve connecting the points $\mathrm{P}_{1}$ and $\mathrm{P}_{2}$ on the triaxial ellipsoid and azimuths $\alpha_{12}$ and $\alpha_{21}$ are desired.

Because of symmetry, the solution of basic geodetic problems on the rotational ellipsoid is relatively simple in mathematical terms. The solution of the basic geodetic problems on the triaxial ellipsoid was a difficult "unsolved" problem in the first half of the 19th century, due to the fact that the motion of the geodesics was not a definite constant. In Jacobi's (1839) work he solved this problem by discovering a constant that allowed this problem to be reduced to a quadratic form. (URL-1, URL-2)

(Panou 2013) presents a method for solving the second problem on the triaxial ellipsoid by directly integrating the ordinary differential equation system for geodesics without using Jacobi's solution.

When we look at the studies

\begin{abstract}
We aim to show how geodetic basic problems will be solved with the modified basic problems. In this paper we study basic geodetic problems (Modified Direct and Invers problem) on a triaxial ellipsoid and we will see how to solve the conversion between the geographical coordinates with Cartesian coordinates or vice versa on triaxial ellipsoid.
\end{abstract}

Key words:Triaxial ellipsoid, Modified Direct and Invers problem, Coordinate transformation

Published on 30th Sept,2017 
related to the subject, it seems that the solution of the direct and invers geodetic problems with geographical coordinates on the triaxial ellipsoid is very difficult. In order to overcome this difficulty, in this work, we made the direct and invers problem with Cartesian coordinates instead of geographical coordinates. We will also use $d_{12}$ slope length that directly connects two points instead of the $\mathrm{S}_{12}$ geodesic curve length. We think that our choice is also meaningful at the same time. This is because the $\mathrm{S}_{12}$ geodesic curve lengths can not be measured with the measuring instruments and can not be applied to the ground, while the slope lengths can easily be measured with modern measuring instruments and can applied to the surface.

So we have a relatively easy solution to the problem. On the other hand, the ability to perform transformation calculations between Cartesian coordinates and geographical coordinates gives us the ability to find the direct and invers geodetic problems results on the triaxial ellipsoid in geographical coordinates from the the direct and invers geodetic problems calculations we have made with Cartesian coordinates.

Although Triaxial ellipsoid equation is quite simple and smooth but geodetic computations are quite difficult on the Triaxial ellipsoid. The main reason for this difficulty is the lack of symmetry.
Triaxial ellipsoid generally not used in geodetic applications. Rotational ellipsoid is frequently used in geodetic applications. Triaxial ellipsoid is although a more general surface so far but has not been used in geodetic applications. The reason for this is not provided as a practical benefit in the calculations. We think this traditional thought ought to be revised again.

Geodetic research has traditionally been motivated by the need to approximate closer and closer the physical reality. Several investigations have shown that the Earth is approximated better by a triaxial ellipsoid rather than a biaxial one. (Bektaş, 2015a).

\section{Which reference surface?}

Geodesic studies need to accept a model for the earth. This model can not be the intersection of the atmosphere with the earth. The oceans show a partial regularity, but we can not talk of such a scheme on land. The reference surface for the earth to be selected according to the actual shape of the earth, measurement and calculation must be easy to connect with it. The most suitable physical model for the earth is geoid. But geoid is not a suitable surface for geodetic computations. Because geoid has undulating shape, has no symmetry and can not be described as math. Therefore reference surface is selected as appropriate geometric surfaces to Earth. Selection of the reference surface depends on the size of the region study and desired accuracy.

The size of the working area and the desired accuracy level determines the reference surface. Triaxial ellipsoid can be evaluated as a more appropriate geometric approach to the geoid according to the rotational ellipsoid. largest semi-axis of a triaxial ellipsoid to be referred to for the Earth is on $345^{\circ}$ longitude and the difference between the equatorial semi-axis is approximately $70 \mathrm{~m}$. (Muller 1991), (Bursa 1995). This 70m difference is not a size that can be ignored in a geodetic sense. On the other hand, there are objections against taking the triaxial ellipsoid as a reference surface physically:

(Torge 2001) The use of triaxial ellipsoid does not significantly reduce the deviations between the geoid and the level ellipsoid. Calculations on these surfaces make the gravitational fields more complex. Finally, they are not suitable as physical normal numbers. For example, although the triaxial rotational ellipsoids are available as equilibrium shapes (homogeneous ellipsoids of Jacobi), such an ellipsoid gives an entirely unnatural form when the actual values are used for the angular velocity and mass of the earth.

Although the triaxial ellipsoid model is not used in geodetic applications in the world today, it is hoped that triaxial ellipsoid or more developed models will be used in the near future modeling of other 
celestial objects, satellites, astreoids and comets, as well as applications such as image processing, face recognition, computer games etc ( Iz et al 2011), (Nyrtsov, et al., 2005).

The reference surfaces that can be selected for the earth are simply ordered towards complexity: Plane (tangential), Sphere, Rotational Ellipsoid and Triaxial ellipsoid (figure-1)
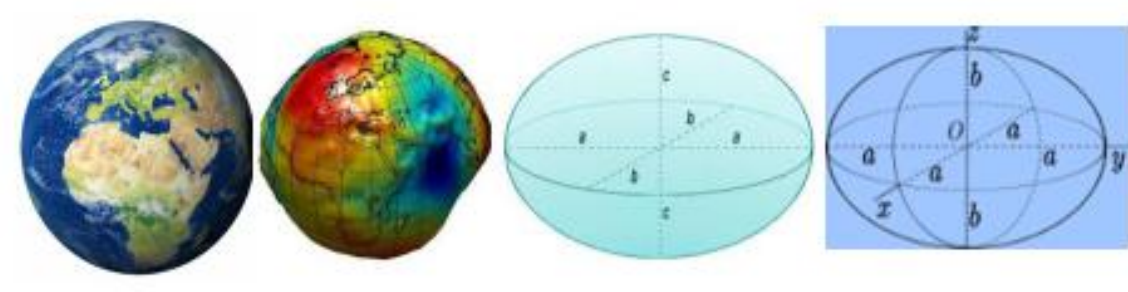

Earth

Sphere

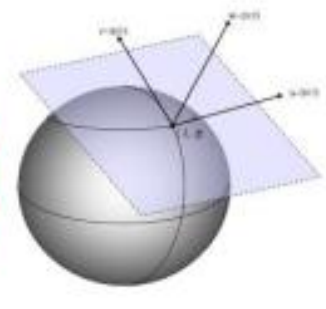

Figure-1: Reference surfaces that can be selected for the Earth

The reference ellipsoids are used as a preferred surface on which geodetic network computations are performed and point coordinates such as latitude, longitude, and elevation are defined.The reference surfaces can be selected for the
Earth from simple to complex: Plane(tangent),Sphere,Ellipsoid (biaxial) and Triaxial ellipsoid

Ease of Calculation

Geoid $>>$ Triaxial Ellipsoid $>>$ Biaxial Ellipsoid $>>$ Sphere $>>$ Tangent Plane

\section{Reality-Accuracy}

If we think of asking a question about the above ranking accuracy computations will be difficult. If desire to simplify on the computational, the accuracy will be reduced. Considering today's computing facilities can not talk about the difficulty computing.

In addition, triaxial ellipsoid surface is most suitable reference surface for Earth and triaxial ellipsoid is more general surface than the rotational ellipsoid. Triaxial ellipsoid formulas are quite useful, because obtaining the rotational ellipsoid formula from triaxial ellipsoid formula is easy. For this, equatorial semi-axis are accepted equal to each other $\left(a_{x}=a_{y}\right.$ $=a)$ which is sufficient on triaxial ellipsoid formula. Similarly to obtain sphere formula from rotational ellipsoid formula it is sufficient to take as $(a=b=R)$. And to obtain plain formula from sphere Formula it is sufficient to take as $(\mathrm{R}=\infty)$ is sufficient (Bektas 2016).
According to common thought of today, biaxial ellipsoid has two different semi-axis which are most suitable for geometric models to the world in geodetic applications. Geodetic applications are generally rotational elipsoid and are use instead of triaxial ellipsoid which will be enough for today's needs.

Geodetic applications in the near future will be used for triaxial ellipsoid or more advanced surfaces are expected. Today, even if triaxial ellipsoid is not use for the Earth, we think it will be use for 
other celestial bodies and other aplications such as; image processing, face recognition, computer games etc. The celestial bodies whose shapes are different from a sphere or ellipsoid it is possible to use the well known triaxial ellipsoid as a reference surface which is mathematically calculated and shows the elongated figure of a body. Many small bodies such as satellites, asteroids or nuclei of comets have more complex figures than a triaxial ellipsoid can show. In accordance $\frac{x^{2}}{a_{x}^{2}}+\frac{y^{2}}{a_{y}^{2}}+\frac{z^{2}}{b^{2}}=1$

The following definitions will be used.

In the Triaxial ellipsoid, the geographical coordinates of a point $(\mathrm{B}, \mathrm{L}, \mathrm{h})$ are determined the surface normal at that point, just as it is in the rotational ellipsoid. The angle between the equatorial plane and Po surface normal is called the ellipsoidal latitude (B), the angle of investigations of Academician Lyapunov A.N. "The celestial bodies have most stable shapes close to triaxial ellipsoid". Traditionally most non-spherical bodies are approximated by a triaxial ellipsoid which is mathematically calculated and shows the non-spherical figure of the body (Nyrtsov, et al., 2005).

\section{.2.1 Triaxial Elipsoid (Elipsoid)}

An ellipsoid is a closed quadric surface that is analogue of an ellipse. Ellipsoid has three different $a_{x}=$ equatorial semimajor axis of the ellipsoid

$a_{y}=$ equatorial semiminor axis of the ellipsoid

$b=$ polar semi-minor axis of the ellipsoid

$\mathrm{L}=$ geodetic longitude

between the meridian plane containing the surface normal at the Po point and the initial (Greenwich) meridian plane is called the ellipsoidal longitude (L) and the distance between the $\mathrm{P}$ landmark and its projection Po point is the ellipsoidal height . The triaxial ellipsoidal surface normals every axes $\left(a_{x}>a_{y}>b\right)$ as shown in Fig.2. Mathematical literature often uses "ellipsoid" in place of "Triaxial ellipsoid or general ellipsoid". Scientific literature (particularly geodesy) often uses "ellipsoid" in place of "biaxial ellipsoid,rotational ellipsoid or ellipsoid revolution". Older literature uses 'spheroid' in place of rotational ellipsoid. The standart equation of an ellipsoid centered at the origin of a cartesian coordinate system and aligned with the axes.

$\mathrm{B}=$ geodetic latitude

$h=$ ellipsoid height

$e_{x}^{2}=\left(a_{x}^{2}-b^{2}\right) / a_{x}^{2} \quad$ first polar eccentricity

$e_{e}^{2}=\left(a_{x}^{2}-a_{y}^{2}\right) / a_{x}^{2}$ first

equatorial eccentricity

time do not cut the $\mathrm{Z}$ axis unlike the rotation ellipsoid.

The local polar measurements $(\mathrm{t}, \beta, \mathrm{d})$ are showing respectively horizontal direction (bearing angle), vertical angle, and slope length measurements between $\mathrm{P}$ and $\mathrm{Q}$ point. 


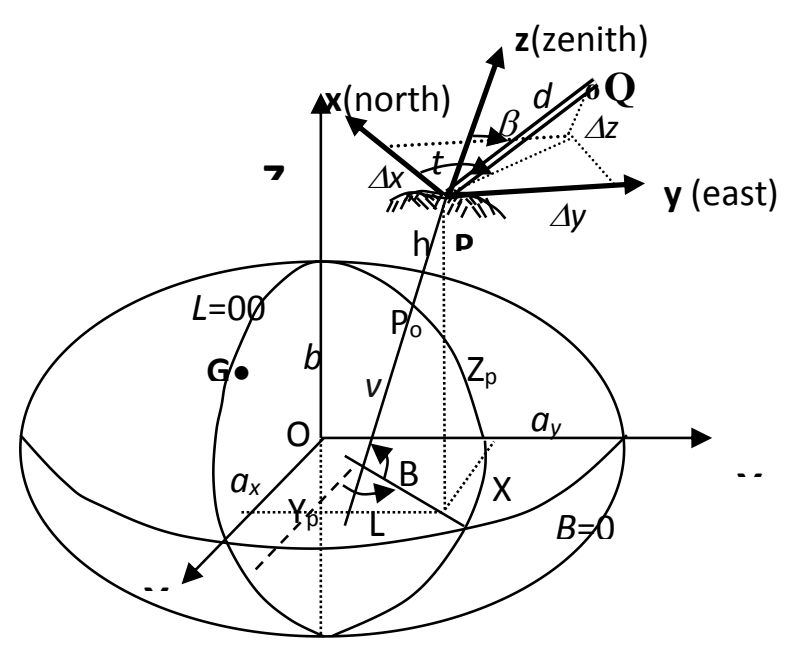

Figure 2: (X Y Z) Cartesian (Global) coordinate system, (B L h) Triaxial ellipsoidal geographical coordinate system and ( $x$ y $z$ ) Local three-dimensional coordinate system and $(t \beta d)$ local polar coordinate system

\subsection{Transformation Between}

Cartesian And Geographical

Coordinates On Triaxial
Ellipsoid

The problem of coordinate transformation is one of the most important issues in the geodetic calculations on the triaxial ellipsoid. The problem of the transformation from Cartesian to geodetic coordinates on triaxial ellipsoid were discussed by several authors ; The problem has been recently discussed by (Feltens, 2009), (Ligas, 2012)

In this study, The details of the Transformation between Cartesian and Geographic Coordinates will not be entered on the Triaxial ellipsoid. For Transformation details (Bektaş, 2014), (Feltens, 2009), (Ligas, 2012) sources can be seen. For example, the Cartesian coordinates from Geographical coordinates are relatively easy and can be performed as follows (Figure-2).

$$
\begin{aligned}
& (\mathrm{B}, \mathrm{L}, \mathrm{h}) \rightarrow(\mathrm{X}, \mathrm{Y}, \mathrm{Z}) \\
& \mathrm{X}=(\mathrm{v}+\mathrm{h}) \cos \mathrm{B} \cos \mathrm{L}
\end{aligned}
$$

$\mathrm{Y}=\left(\mathrm{v} .\left(1-e_{e}^{2}\right)+\mathrm{h}\right) \cos \mathrm{B} \sin \mathrm{L}$

$\mathrm{Z}=\left(\mathrm{v} \cdot\left(1-e_{x}^{2}\right)+\mathrm{h} \cdot \operatorname{Sin} \mathrm{B}\right.$

$$
v=\frac{a_{x}}{\sqrt{1-e_{x}^{2} \sin ^{2} B-e_{e}^{2} \cos ^{2} B \sin ^{2} L}}
$$

$v=$ radius of main curvature

The following link can be used for calculations

http://www.mathworks.com/matlab central/fileexchange/46239-convert er-geodetic-coordinates-to-cartesia n-coordinates

$(\mathrm{X}, \mathrm{Y}, \mathrm{Z}) \rightarrow(\mathrm{B}, \mathrm{L}, \mathrm{h})$ transformation shown in the referenrces section, to make transformation calculations on the triaxial ellipsoid between the
Cartesian coordinates and the geographical coordinates.

The following link can be used for calculations

http://www.mathworks.com/matlab central/fileexchange/46248-convert er-cartesian-coordinates-to-geodeti c-coordinates

\section{Three-Dimensional Coordinate Transformation}

As it is known, 7 transformations (datum) parameters must be known in order to convert between two three dimensional coordinate systems. The 7 datum parameters are: 3 shifted parameters (tx, ty, tz), 3 rotation parameters ( $\varepsilon x, \varepsilon y, \varepsilon z$ ) and 1 scale $(\mathrm{k})$ parameters. These parameters are determined as coordinates of at least 2 points in both coordinate systems and only one coordinate of the other point is known. If the coordinates of three or more points are known in both systems, the transformations 
parameters are determined by We can do similarity coordinate systems (XYZ and xyz) adjustement (Bektaş, 2005).

transformation between two without scale factor as follows. $\mathrm{R}_{3 \times 3}$ three-dimensional Cartesian rotation matrix (Eq.6);

$R=\left[\begin{array}{ccc}\cos \varepsilon_{\mathrm{y}} \cos \varepsilon_{\mathrm{z}} & \cos \varepsilon_{\mathrm{x}} \sin \varepsilon_{\mathrm{z}}+\sin \varepsilon_{\mathrm{x}} \sin \varepsilon_{\mathrm{y}} \cos \varepsilon_{\mathrm{z}} & \sin \varepsilon_{\mathrm{x}} \sin \varepsilon_{\mathrm{z}}-\cos \varepsilon_{\mathrm{x}} \sin \varepsilon_{\mathrm{y}} \cos \varepsilon_{\mathrm{z}} \\ -\cos \varepsilon_{\mathrm{y}} \sin \varepsilon_{\mathrm{z}} & \cos \varepsilon_{\mathrm{x}} \cos \varepsilon_{\mathrm{z}}-\sin \varepsilon_{\mathrm{x}} \sin \varepsilon_{\mathrm{y}} \sin \varepsilon_{\mathrm{z}} & \sin \varepsilon_{\mathrm{x}} \cos \varepsilon_{\mathrm{z}}+\cos \varepsilon_{\mathrm{x}} \sin \varepsilon_{\mathrm{y}} \sin \varepsilon_{\mathrm{z}} \\ \sin \varepsilon_{\mathrm{y}} & -\sin \varepsilon_{\mathrm{x}} \cos \varepsilon_{\mathrm{y}} & \cos \varepsilon_{\mathrm{x}} \cos \varepsilon_{\mathrm{y}}\end{array}\right]$

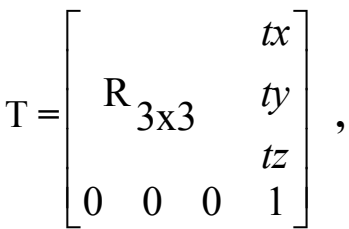

Three-Dimensional Coordinate Transformation with $\mathrm{T}$ The expanded transformation matrix can be easily transformed from xyz to $\mathrm{XYZ}$ or vice versa as in (Eq.7) (Bektas, 2015a).

\subsection{Transformations Between} Global (XYZ) and Local Vertical Coordinate System (xyz)

Three dimensional (3D) geodesy naturally uses three dimensional coordinate systems. From these coordinate systems, the three-dimensional local coordinate system $\mathrm{xyz}$ and the global coordinate system (geocentric) system XYZ are widely used. As it is known, the three dimensional local coordinate system $(\mathrm{x}, \mathrm{y}, \mathrm{z})$ can be used in areas where the work area is not very large. The center point of the

$$
\left[\begin{array}{c}
X_{i} \\
Y_{i} \\
Z_{i} \\
1
\end{array}\right]=\mathbf{T}\left[\begin{array}{c}
x_{i} \\
y_{i} \\
z_{i} \\
1
\end{array}\right]
$$$$
\left[\begin{array}{c}
x_{i} \\
y_{i} \\
z_{i} \\
1
\end{array}\right]=\mathbf{T}^{-1}\left[\begin{array}{c}
X_{i} \\
Y_{i} \\
Z_{i} \\
1
\end{array}\right]
$$

system is usually chosen in the middle of the study area (Öztürk and Şerbetçi 1989).

The xyz local (topocentric) system used here is a three-dimensional model coordinate system, the $\mathrm{z}$-axis is containing ellipsoid's surface normal on $\mathrm{P}$ point. However, the coordinate system in which the measurements are made is the toposentric coordinate system at point $\mathrm{P}$, which coincides with the tangent to the geoid normal of the plumb curve passing through the $\mathrm{Z}$-axis $\mathrm{P}$ land point. Making the measurements in the natural system and the calculations in the model system will cause the fault. In this case there are two operations that need to be done. The first one is to accept the error, that is, to assume that the ellipsoid normal coincides with the geoid normal (the plumb line) at that point. The second and, in fact, the reduction of the $t$ horizontal direction (bearing angle) and $\beta \quad$ vertical angle measurements made at point $\mathrm{P}$ to the model system with the help of the plumb line components at point P. In other words, to convert the measurements from the geoid normal to the ellipsoid normal (Moritz, 1980). The direction of the $\mathrm{x}$ axis is towards the $\mathrm{Z}$ axis (north pole point) and is called north. $y$ axis was oriented to the east and formed a left-handed system (figure-3). The XYZ global coordinate system (figure-2) is a right-handed system.

We know that seven datum parameters are needed to convert the similarity between two three dimensional coordinate systems. If there is no scale difference between $\mathrm{XYZ}$ and $\mathrm{xyz}$, the required number of conversion parameters is 
six. If we know the coordinates of the origin ( $\mathrm{P}$ point) of the local orthogonal coordinate system, We have determined three shift parameters required for the transformation. The rotation parameter $\left(\varepsilon_{\mathrm{X}}, \varepsilon_{\mathrm{Y}}, \varepsilon_{\mathrm{Z}}\right)$ three around the $\mathrm{x}, \mathrm{y}, \mathrm{z}$ axes to be determined is

$\mathbf{x}=\left[\begin{array}{l}x_{Q}-x_{P} \\ y_{Q}-y_{P} \\ z_{Q}-z_{P}\end{array}\right] \quad \mathbf{X}=\left[\begin{array}{c}X_{Q}-X_{P} \\ Y_{Q}-Y_{P} \\ Z_{Q}-Z_{P}\end{array}\right]$

$\mathrm{x}$ Local coordinate differences, $\mathrm{X}$ global coordinate differences

$\mathbf{R}^{\mathbf{T}}=\left[\begin{array}{ccc}-\sin B_{P} \cos L_{P} & -\sin B_{P} \sin L_{P} & \cos B_{P} \\ -\sin L_{P} & \cos L_{P} & 0 \\ \cos B_{P} \cos L_{P} & \cos B_{P} \sin L_{P} & \sin B_{P}\end{array}\right]$

$\mathbf{R}$ ortogonal transformation matrix $\left(\mathrm{R}^{\mathrm{T}}=\mathrm{R}^{-1}\right)$

Local coordinate differences from global coordinate differences

Using the orthogonal transformation matrix $\quad\left(\mathrm{R}^{\mathrm{T}}=\mathrm{R}^{-1}\right)$

- Local coordinate differences from global coordinate differences

$\mathrm{x}=\mathrm{R}^{\mathrm{T}} \cdot \mathrm{X}$,

- Global coordinate differences from local coordinate differences $X=R \cdot x$.

4. Geodetic Computations With Three-Dimensional (3D) easily obtained from the geographical coordinates of the origin

point $\mathrm{P}\left(\varepsilon_{\mathrm{X}}=0, \varepsilon_{\mathrm{Y}}=90^{\circ}-\mathrm{B}, \varepsilon_{\mathrm{Z}}=180^{\circ}-\mathrm{L}\right) . \quad$ In summary, only the Cartesian (XYZ) or (BLh) geographical coordinates of the $\mathrm{P}$ origin point are sufficient

The $\mathrm{R}_{3 \times 3}$ transformation matrix is obtained from the geographical to transformation between $\mathrm{XYZ}$ and xyz coordinate systems.

Transformations xyz local, XYZ global coordinates are performed from the following equations on between $\mathrm{P}$ and $\mathrm{Q}$ points (Öztürk and Şerbetçi 1989).

coordinates of the origin point as follows.
The direct and invers problem calculations that can be done on the plane, sphere and ellipsoid surface can also be performed on 3D Cartesian coordinates. Nowadays, we see that applications of positioning and surveying with GPS receivers are increasing day by day. We know that GPS receivers also produce Cartesian coordinates as raw, and other coordinate types (geographical, UTM, etc.) are derived from these Cartesian coordinates. It is clear that errors resulting from the transformation during the derivation phase will also affect the coordinates. Therefore, it is evaluated that working with raw Cartesian coordinates is more appropriate in terms of accuracy. On the other hand, we will be able to find the direct and invers problem calculations from the ellipsoid and sphere surface with Geographical coordinates from the direct and invers problem calculations that we will do with Cartesian coordinates.

\subsection{Transformations Between} Local Polar Coordinates $(t, \boldsymbol{\beta}, \mathbf{d})$ and Local Orthogonal Coordinates $(x, y, z)$

$\Delta \mathrm{x}, \Delta \mathrm{y}$ and $\Delta \mathrm{z}$ are showing coordinate differences between the two points. These coordinate differences are obtained from the polar coordinate $(t, \beta, d)$ following equations (Figure-3). 
$\Delta \mathrm{x}=\mathrm{d} \sin \beta \cos \mathrm{t} \quad \mathrm{d}=\left(\Delta \mathrm{x}^{2}+\Delta \mathrm{y}^{2}+\Delta \mathrm{z}^{2}\right)^{1 / 2} \quad$ : slope distance

$\Delta \mathrm{y}=\mathrm{d} \sin \beta \sin \mathrm{t} \quad \mathrm{t}=\arctan (\Delta \mathrm{y} / \Delta \mathrm{x}) \quad: \quad$ bearings (horizontal direction)

$\Delta \mathrm{z}=\mathrm{d} \cos \beta \quad \beta=\arccos (\Delta \mathrm{z} / \mathrm{d}) \quad$ : vertical angle

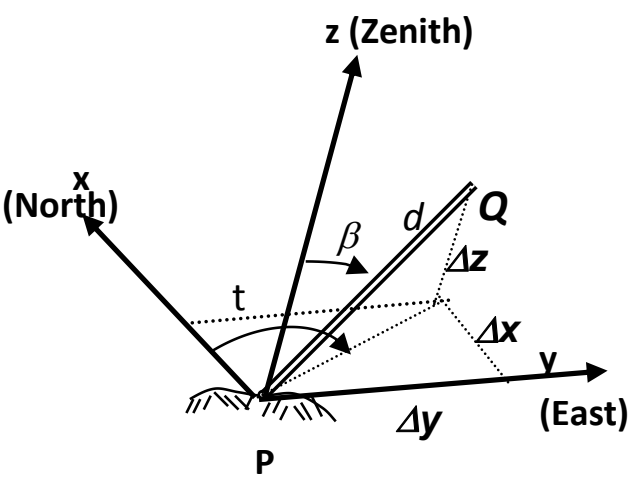

Figure-3. Polar coordinates $(t, \beta, d)$ and local orthogonal coordinates $(x, y, z)$

\subsection{The Polar Coordinates From}

The Global Coordinates,
The Polar coordinates $(t, \beta, d)$ between the two points are obtained from the transformation matrix $\mathrm{R}$ as follows. $\Delta \mathrm{X}, \Delta \mathrm{Y}$ ve $\Delta \mathrm{Z}$ are showing the global orthogonal coordinate differences between two points

$$
\begin{gathered}
d=\sqrt{\Delta X^{2}+\Delta Y^{2}+\Delta Z^{2}} \\
t=\arctan \left(\frac{-\Delta X \sin \Lambda+\Delta Y \cos \Lambda}{-\Delta X \sin \Phi \cos \Lambda-\Delta Y \sin \Lambda \sin \Phi+\Delta Z \cos \Phi}\right) \\
\beta=\operatorname{arc~cos}\left(\frac{\Delta X \cos \Phi \cos \Lambda+\Delta Y \cos \Phi \sin \Lambda+\Delta Z \sin \Phi}{\sqrt{\Delta X^{2}+\Delta Y^{2}+\Delta Z^{2}}}\right)
\end{gathered}
$$

In these formulas, the values $\Phi, \Lambda$ are the astronomical latitude and longitude values of the $\mathrm{P}$ origin point. However, it is difficult to obtain these values. For this reason, instead of $\Phi, \Lambda$ approximately ellipsoidal B, L values are used in practice. If the components of the plumb line $\Lambda=L+\eta \sec B$ deviation $(\xi, \eta)$ are known at point P. 
Therefore above equation can be used (Moritz, 1980). How to calculate the ellipsoidal $\mathrm{B}, \mathrm{L}$ values from the point of the global (XYZ) coordinates has already been mentioned in section 2.2

\subsection{Modified Direct Problem} with 3D Cartesian Coordinates

\section{On Triaxial Ellipsoid}

Given: Cartesian coordinates of $\mathrm{P}_{1}$ $\left(\mathrm{X}_{1}, \mathrm{Y}_{1}, \mathrm{Z}_{1}\right)$ and Polar coordinates $t_{12}$ bearing angle, $\beta_{12}$ vertical angle and $d_{12}$ slope distance to $\mathrm{P}_{2}$ point.

$$
\begin{aligned}
\Delta \mathrm{x} & =\mathrm{d} \sin \cos \cos \mathrm{t} \\
\Delta \mathrm{y} & =\mathrm{d} \sin \beta \sin \mathrm{t} \\
\Delta \mathrm{z} & =\mathrm{d} \cos \beta
\end{aligned}
$$

From these differences the global coordinate differences $(\Delta \mathrm{X}, \Delta \mathrm{Y}, \Delta \mathrm{Z})$ are found through the $\mathrm{R}$ transformation matrix. Where the transformation matrix $\mathrm{R}$ is directly

$$
\begin{aligned}
& \mathbf{X}=\left[\begin{array}{l}
\Delta X \\
\Delta Y \\
\Delta Z
\end{array}\right] \quad \mathbf{x}=\left[\begin{array}{c}
\Delta x \\
\Delta y \\
\Delta z
\end{array}\right] \quad \rightarrow\left[\begin{array}{c}
\Delta X \\
\Delta Y \\
\Delta Z
\end{array}\right]=\mathbf{R}\left[\begin{array}{l}
\Delta x \\
\Delta y \\
\Delta z
\end{array}\right] \\
& \mathbf{X}=\mathbf{R} \quad \mathbf{x} \rightarrow \text { coordinates of point } \mathrm{P}_{1} \\
& \text { coordinate } \text { to the } \\
& X_{2}=X_{1}+\Delta X \\
& Y_{2}=Y_{1}+\Delta Y \\
& Z_{2}=Z_{1}+\Delta Z
\end{aligned}
$$

The calculated global coordinate differences are added to the

coordinates of point $\mathrm{P}_{1}\left(\mathrm{X}_{1}, \mathrm{Y}_{1}, \mathrm{Z}_{1}\right)$

dependent the geographical (B, L) coordinates of the $\mathrm{P}$ origin point.

The geographical coordinates depend on the normal of the surface, so the reference surface
Requirements:

Cartesian

coordinates of $\mathrm{P}_{2}\left(\mathrm{X}_{2}, \mathrm{Y}_{2}, \mathrm{Z}_{2}\right)$

Solution of Problem: The local orthogonal coordinate differences between $\mathrm{P}_{1}$ and $\mathrm{P}_{2}$ are found from the given polar coordinates,

(Triaxial, Rotational ellipsoid, Sphere) to be used in solving the problem will change the geographical coordinates of the point.

to find the coordinates of point $\mathrm{P}_{2}\left(\mathrm{X}_{2}, \mathrm{Y}_{2}, \mathrm{Z}_{2}\right)$

\section{Numerical Example-1: Cartesian} coordinates of $\mathrm{P}_{1}$ point
$\left(\mathrm{X}_{\mathrm{P} 1}=4235882.4602\right.$

$\mathrm{Y}_{\mathrm{P} 1}=3554249.4108$
$3171030.2321 \mathrm{~m})$ and Polar coordinates $\mathrm{t}_{12}=30^{\circ}$ bearing angle, 
$\beta \quad{ }_{12}=87^{\circ}$ vertical angle and $\mathrm{d}_{12}=3500 \mathrm{~m}$ slope distance to $\mathrm{P}_{2}$ point.

Solve the problem and find the Cartesian coordinates of $\mathrm{P}_{2}\left(\mathrm{X}_{2}, \mathrm{Y}_{2}, \mathrm{Z}_{2}\right)$ separately on the sphere, ellipsoid and triaxial ellipsoid surfaces.

Use surfaces parameters below

For Triaxial elipsoid $\left(a_{x}=6378388 \mathrm{~m} ; \quad a_{y}=6378318 \mathrm{~m}\right.$; $b=6356911.9461 \mathrm{~m})$
For Rotational ellipsoid $\Delta \mathrm{y}=d \sin \beta \sin \mathrm{t} \quad=1747.6017 \mathrm{~m}$ $(a=6378388 \mathrm{~m}$; $b=6356911.94613 \mathrm{~m})$

For Sphere $(\mathrm{R}=6374000 \mathrm{~m})$

\section{Solution of the problem:}

First the local orthogonal coordinate differences between $\mathrm{P}_{1}$ and $\mathrm{P}_{2}$ are found from the given polar coordinates,

$\Delta \mathrm{x}=d \sin \beta \cos \mathrm{t}=3026.9349 \mathrm{~m}$
$\Delta \mathrm{z}=d \cos \beta$ $=183.1758 \mathrm{~m}$

The calculation of the geographic coordinates of point $\mathrm{P}_{1}$ from the cartesian coordinates, this step depends on the reference surface selected (URL-1)

Use of MATLAB function

$$
\begin{gathered}
\text { [Geo] = cartesian_geodetic( [XP1 YP1 ZP1 } \left.], \quad\left[\begin{array}{lll}
\text { ax } & \text { ay } & \text { b }
\end{array}\right]\right) \quad \text { For triaxial ellipsoid } \\
\mathrm{B}_{1}=30^{\circ} \quad \text { ve } \quad \mathrm{L}_{1}=40^{\circ} \quad \mathrm{h}_{1}=1200.0000
\end{gathered}
$$

$[\mathrm{Geo}]=$ cartesian_geodetic $\left([\mathrm{XP1}\right.$ YP1 ZP1 $\left.], \quad\left[\begin{array}{lll}\mathrm{a} & \mathrm{a} & \mathrm{b}\end{array}\right]\right) \quad$ For rotational ellipsoid

$$
\mathrm{B}_{1}=30^{\circ} 0^{\prime} 0.8119^{\prime} \quad \mathrm{L}_{1}=39^{\circ} 59^{\prime} 57.7711^{\prime \prime} \mathrm{h}_{1}=1178.2904 \mathrm{~m}
$$

$[\mathrm{Geo}]=$ cartesian_geodetic $\left([\mathrm{XP1}\right.$ YP1 ZP1 $\left.], \quad\left[\begin{array}{lll}\mathrm{a} & \mathrm{a} & \mathrm{a}\end{array}\right]\right) \quad$ For sphere

$$
\mathrm{B}_{1}=29^{\circ} 49^{\prime} 59.4740^{\prime} \quad \mathrm{L}_{1}=39^{\circ} 59^{\prime} 57.771^{\prime \prime} \quad \mathrm{h}_{1}=231.0771 \mathrm{~m}
$$

From these geographical

matrix is established as follows (9) and the Global orthogonal coordinates, the $\mathrm{R}$ transformation coordinate differences;

$$
R^{T}=\left[\begin{array}{ccc}
-0.38302 & -0.32139 & 0.86602 \\
-0.64278 & 0.76604 & 0 \\
0.66341 & 0.55667 & 0.5000
\end{array}\right] \text { for triaxial elipsoid }
$$

$$
\left[\begin{array}{lll}
\Delta \mathrm{X} & \Delta \mathrm{Y} & \Delta \mathrm{Z}
\end{array}\right]^{\mathrm{T}}=\mathbf{R}\left[\begin{array}{lll}
\Delta \mathrm{x} & \Delta \mathrm{y} & \Delta \mathrm{z}
\end{array}\right]^{\mathrm{T}}=\quad\left[\begin{array}{lll}
-2161.1986 & 467.8710 & 2712.9905
\end{array}\right]^{\mathrm{T}}
$$

Cartesian coordinates of $\mathrm{YP}_{2}=3554717.2818$

$[\mathrm{Geo}]=$ cartesian_geodetic $([\mathrm{XP} 2$

$\mathrm{P}_{2}\left(\mathrm{X}_{2}, \mathrm{Y}_{2}, \mathrm{Z}_{2}\right)$

$$
\mathrm{ZP}_{2}=3173743.2226
$$

$$
\begin{aligned}
X_{2} & =X_{1}+\Delta X \\
Y_{2} & =Y_{1}+\Delta Y \\
Z_{2} & =Z_{1}+\Delta Z
\end{aligned}
$$

$\mathrm{XP}_{2}=4233721.2616 \mathrm{~m}$

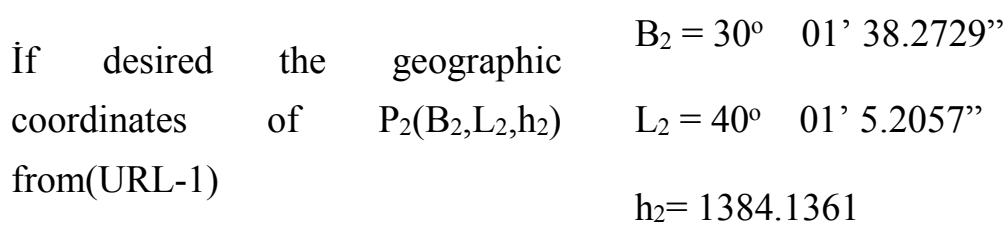

If desired the geographic

1384.136 
The results of the direct problem on the surface of the rotation ellipsoid and sphere are given in Table 1

below.

\begin{tabular}{|c|c|c|c|}
\hline & $\mathrm{B}_{1}, \mathrm{~L}_{1}, \mathrm{~h}_{1}$ & $\mathrm{X}_{2}, \mathrm{Y}_{2}, \mathrm{Z}_{2}$ & $\mathrm{~B}_{2}, \mathrm{~L}_{2}, \mathrm{~h}_{2}$ \\
\hline Triaxial ellipsoid & $\begin{array}{c}\mathrm{B}_{1}=30^{\circ} \\
\mathrm{L}_{1}=40^{\circ} \\
\mathrm{h}_{1}=1200.0000\end{array}$ & $\begin{array}{l}X_{2}=4233721.2616 \\
Y_{2}=3554717.2818 \\
Z_{2}=3173743.2226\end{array}$ & $\begin{array}{c}\mathrm{B}_{2}=30^{\circ} 01^{\prime} 38.2729^{\prime} \\
\mathrm{L}_{2}=40^{\circ} 01^{\prime} 5.2057^{\prime} \\
\mathrm{h}_{2}=1384.1361\end{array}$ \\
\hline Rotational ellipsoid & $\begin{array}{c}\mathrm{B}_{1}=30^{\circ} 0^{\prime} 0.8119^{\prime \prime} \\
\mathrm{L}_{1}=39^{\circ} 59^{\prime} 57.7711^{\prime \prime} \\
\mathrm{h}_{1}=1178.2904 \mathrm{~m}\end{array}$ & $\begin{array}{c}\mathrm{X}_{2}=4233721.2584 \mathrm{~m} \\
\mathrm{Y}_{2}=3554717.2983 \\
\mathrm{Z}_{2}=3173743.2172\end{array}$ & $\begin{array}{c}\mathrm{B}_{2}=30^{\circ} 01^{\prime} 39.08559^{\prime \prime} \\
\mathrm{L}_{2}=40^{\circ} \quad 01^{\prime} 2.9772^{\prime \prime} \\
\mathrm{h}_{2}=1362.4266 \mathrm{~m}\end{array}$ \\
\hline Sphere & $\begin{array}{c}\mathrm{B}_{1}=29^{\circ} 49^{\prime} 59.4740^{\prime \prime} \\
\mathrm{L}_{1}=39^{\circ} 59^{\prime} 57.771^{\prime} \\
\mathrm{h}_{1}=231.0771 \mathrm{~m}\end{array}$ & $\begin{array}{c}\mathrm{X}_{2}=4233727.3218 \mathrm{~m} \\
\mathrm{Y}_{2}=3554722.3860 \\
\mathrm{Z}_{2}=3173747.1556\end{array}$ & $\begin{array}{c}\mathrm{B}_{2}=29^{\circ} \quad 51^{\prime} 37.4158^{\prime \prime} \\
\mathrm{L}_{2}=40^{\circ} \quad 01^{\prime} 2.9771^{\prime \prime} \\
\mathrm{h}_{2}=415.2111\end{array}$ \\
\hline
\end{tabular}

Table 1 The results of the direct problem on triaxial ellipsoid, rotational ellipsoid and sphere.

\subsection{Modified Invers problem with 3D Cartesian Coordinates} On Triaxial Ellipsoid

Given: Cartesian coordinates of $\mathrm{P}_{1}$ $\left(\mathrm{X}_{1}, \mathrm{Y}_{1}, \mathrm{Z}_{1}\right)$ and $\mathrm{P}_{2}\left(\mathrm{X}_{2}, \mathrm{Y}_{2}, \mathrm{Z}_{2}\right)$ are given.

Requirements: Polar coordinates $t_{12}$ bearing angle, $\beta_{12}$ vertical angle and $d_{12}$ slope distance between $\mathrm{P}_{1}$ and $\mathrm{P}_{2}$ point

Solution: First the geographical coordinates of point $\mathrm{P}_{1}(\mathrm{~B}, \mathrm{~L})$ are found from the Cartesian coordinates of $\mathrm{P}_{1}\left(\mathrm{X}_{1}, \mathrm{Y}_{1}, \mathrm{Z}_{1}\right)$ by (URL-1)
After that Global Cartesian coordinate differences are found as below

$$
\begin{gathered}
\Delta \mathrm{X}=\mathrm{X}_{2}-\mathrm{X}_{1} \quad \Delta \mathrm{Y}=\mathrm{Y}_{2}-\mathrm{Y}_{1} \\
\Delta \mathrm{Z}=\mathrm{Z}_{2}-\mathrm{Z}_{1}
\end{gathered}
$$

The desired polar coordinates $\left(t_{12}\right.$ bearing angle , $\beta_{12}$ vertical angle and $d_{12}$ slope distance) between $\mathrm{P}_{1}$ and $\mathrm{P}_{2}$ point are found from equations (11), (12), (13).

\section{Numerical Example-2:}

Given: Cartesian coordinates of $\mathrm{P}_{1}$ $\left(\mathrm{X}_{1}, \mathrm{Y}_{1}, \mathrm{Z}_{1}\right)$ and $\mathrm{P}_{2}\left(\mathrm{X}_{2}, \mathrm{Y}_{2}, \mathrm{Z}_{2}\right)$

$\mathrm{XP}_{1}=4235882.4602 \quad \mathrm{YP}_{1}=$
$3554249.4108 \quad \mathrm{ZP}_{1}=$
3171030.2321
$\mathrm{XP}_{2}=\quad 4233721.2616$
$\mathrm{YP}_{2}=3554717.2818 \mathrm{ZP}_{2}=$
3173743.2226

Requirements: Polar coordinates $t_{12}$ bearing angle , $\beta_{12}$ vertical angle and $d_{12}$ slope distance between $\mathrm{P}_{1}$ and $\mathrm{P}_{2}$ point

Solution: First the geographical coordinates of point $\mathrm{P}_{1}\left(\mathrm{~B}_{1}, \mathrm{~L}_{1}\right)$ are found from the Cartesian coordinates $\mathrm{P}_{1}\left(\mathrm{X}_{1}, \mathrm{Y}_{1}, \mathrm{Z}_{1}\right)$ by (URL-1) 
$[\mathrm{Geo}]=$ cartesian_geodetic([XP1 YP1 ZP1], [ax ay b])

$\mathrm{B}_{1}=30^{\circ} \quad$ ve $\mathrm{L}_{1}=40^{\circ} \quad \mathrm{h}_{1}=$ 1200.0000
After that Global Cartesian coordinate differences are found as below

$$
\begin{gathered}
\Delta \mathrm{X}=\mathrm{X}_{2}-\mathrm{X}_{1}=-2161.1986 \\
\Delta \mathrm{Y}=\mathrm{Y}_{2}-\mathrm{Y}_{1}=467.8710 \\
\Delta \mathrm{Z}=\mathrm{Z}_{2}-\mathrm{Z}_{1}=2712.9905
\end{gathered}
$$

The desired polar coordinates( $t_{12}$ bearing angle,$\beta_{12}$ vertical angle and $d_{12}$ slope distance) between $\mathrm{P}_{1}$ and $\mathrm{P}_{2}$ point are found from equations (11), (12), (13).

$d=\sqrt{\Delta X^{2}+\Delta Y^{2}+\Delta Z^{2}}=3500 \mathrm{~m}$

$t=\arctan \left(\frac{-\Delta X \sin \Lambda+\Delta Y \cos \Lambda}{-\Delta X \sin \Phi \cos \Lambda-\Delta Y \sin \Lambda \sin \Phi+\Delta Z \cos \Phi}\right)=30^{\circ}$

$\beta=\arccos \left(\frac{\Delta X \cos \Phi \cos \Lambda+\Delta Y \cos \Phi \sin \Lambda+\Delta Z \sin \Phi}{\sqrt{\Delta X^{2}+\Delta Y^{2}+\Delta Z^{2}}}\right)=87^{\circ}$

The results of the Invers problem ellipsoid and sphere are given in

\begin{tabular}{|c|c|c|c|}
\hline & $\mathrm{B}_{1}, \mathrm{~L}_{1}, \mathrm{~h}_{1}$ & $\mathrm{~B}_{2}, \mathrm{~L}_{2}, \mathrm{~h}_{2}$ & $\mathrm{t}_{12}, \beta_{12}, \mathrm{~d}_{12}$, \\
\hline Triaxial ellipsoid & $\begin{array}{l}\mathrm{B}_{1}=30^{\circ} \\
\mathrm{L}_{1}=40^{\circ} \\
\mathrm{h}_{1}=1200.0000\end{array}$ & $\begin{array}{l}\mathrm{B}_{2}=30^{\circ} 1^{\prime} 38.2729^{\prime \prime} \\
\mathrm{L}_{2}=40^{\circ} 01^{\prime} 5.2057^{\prime \prime} \\
\mathrm{h}_{2}=1384.1361\end{array}$ & $\begin{array}{l}\mathrm{t}_{12}=30^{\circ} \\
\beta_{12}=87^{\circ} \\
\mathrm{d}_{12}=3500.0000 \mathrm{~m}\end{array}$ \\
\hline Rotational ellipsoid & $\begin{array}{l}\mathrm{B}_{1}=30^{\circ} 0^{\prime} 0.8119^{\prime \prime} \\
\mathrm{L}_{1}=39^{\circ} 59^{\prime} 57.7711^{\prime \prime} \\
\mathrm{h}_{1}=1178.2904 \mathrm{~m}\end{array}$ & $\begin{array}{l}\mathrm{B}_{2}=30^{\circ} 01^{\prime} 39.0856^{\prime \prime} \\
\mathrm{L}_{2}=40^{\circ} \quad 1^{\prime} 2.9772^{\prime \prime} \\
\mathrm{h}_{2}=1362.4266 \mathrm{~m}\end{array}$ & $\begin{array}{l}\mathrm{t}_{12}=29^{\circ} 59^{\prime} 58.9944^{\prime \prime} \\
\beta_{12}=87^{\circ} 0^{\prime} 0.2620^{\prime \prime} \\
\mathrm{d}_{12}=3500.0000 \mathrm{~m}\end{array}$ \\
\hline Sphere & $\begin{array}{l}\mathrm{B}_{1}=29^{\circ} 49 \quad 59.4740^{\prime \prime} \\
\mathrm{L}_{1}=39^{\circ} 59^{\prime} 57.771^{\prime \prime} \\
\mathrm{h}_{1}=231.0771 \mathrm{~m}\end{array}$ & $\begin{array}{l}\mathrm{B}_{2}=29^{\circ} 51^{\prime} 37.4158^{\prime \prime} \\
\mathrm{L}_{2}=40^{\circ} 01^{\prime} 2.9771^{\prime \prime} \\
\mathrm{h}_{2}=415.2111\end{array}$ & $\begin{array}{l}\mathrm{t}_{12}=29^{\circ} 59^{\prime} 43.6191^{\prime \prime} \\
\beta_{12}=87^{\circ} 08^{\prime} 41.0486^{\prime \prime} \\
d_{12}=3500.0000 \mathrm{~m}\end{array}$ \\
\hline
\end{tabular}
on the surface of the rotation Table 2 below.

Tablo 2 The results of the invers problem on the surface of the triaxial ellipsoid, rotation ellipsoid and sphere 


\section{Application Results}

In this paper, it is shown numerically how to perform modified direct and Invers problem calculations with Cartesian coordinates on triaxial ellipsoid. In addition, the same Direct and Invers problem calculations have been made possible to solve both the rotational ellipsoid and the spherical surface and compare the results. It is very normal to obtain different results on different reference surfaces. Naturally, if the triaxial ellipsoid's equatorial half-axes approach each other; triaxial ellipsoid results will approach the results of Rotational ellipsoid. Likewise, the rotational ellipsoid results will approach the global results as far as the rotational ellipsoid flattening is approaching zero.

\section{Conclusion}

In this study, how to solve the basic geodetic problems (modified direct and invers problem) on the triaxial ellipsoid is discussed. The triaxial ellipsoid is a more general surface than the sphere and rotational ellipsoid surfaces from which the reference surface can be obtained for the Earth. For this reason, Direct and invers problem on the spherical ellipsoid and sphere surfaces were also obtained numerically from the relations of the Direct and invers problem excluded for the triaxial ellipsoid.
The algorithm used for direct and invers problem calculations made on Cartesian coordinates on the triaxial ellipsoid can also be used to make direct and invers problem calculations with geographical coordinates. The only way to do this is to convert the given geographic coordinates into Cartesian coordinates.

\section{References}

[1]Bektas, S,(2016), Pratik Jeodezi- Ölçme Bilgisi, OMÜ yayınları, Samsun

[2]Bektas, S,(2014), Orthogonal Distance From An Ellipsoid, Boletim de Ciencias Geodesicas, Vol. 20, No. 4 ISSN 1982-2170, 2014

[3]Bektaş,S.(2015), Geodetic Computations on Triaxial Ellipsoid, International Journal of Mining Science (IJMS) Volume 1, Issue 1, June 2015, PP 25-34

[4]Feltens J.(2009), Vector method to compute the Cartesian $(X, Y, Z)$ to geodetic $(\varphi, \lambda, h)$ transformation on a triaxial ellipsoid, J Geod 83:129-137.

[5]İz H. B., Ding X. L., Dai C. L. and Shum C. K.(2011), Polyaxial figures of the Moon, J. Geod. Sci., 1, 348-35

[6]Jacobi C. G. J., (1839) Note von der geodätischen linie auf einem ellipsoid und den verschiedenen anwendungen einer merkwürdigen analytischen substitution, J. Crelle, 19, 309-313.

[7]Ligas M.(2012), Cartesian to geodetic coordinates conversion on a triaxial ellipsoid, J. Geod., 86, 249-256.

[8]Moritz, H.(1980), Advanced Physical Geodesy, Herbert Wichmann Verlag Karlsruhe. 1980

[9]Müller $\quad$ B.(1991), Kartenprojektionen des dreiachsigen Ellipsoids, Diploma thesis, supervisor EW Grafarend, http://www.uni-stuttgart.de/ gi/education/ diplomarbeiten/ bmueller.pdf,61 pp (in German)

[10]Nyrtsov M.V.,Bugaevsky L.M.,Stooke, P. J.(2005), The Multiple Axis Ellipsoids As Reference Surfaces For Mapping Of Small Celestial Bodies, Lev M. Bugaevsky, Moscow State University of Geodesy and Cartography (MIIGAiK), Moscow, Russia

[11]Panou (2013), The geodesic boundary value problem and its solution on a triaxial ellipsoid, Journal of Geodetic Science, • $3(3)$ - 2013 - 240-249 DOI: 10.2478/jogs-2013-0028

[12]Torge W. (2001) Geodesy. Third completel $y$ revised and extended edition. Walter de Gruyter · Berlin · New York 